\title{
System of recursive equations for the partition functions of 1D models
}

\author{
U.A. Rozikov ${ }^{1}$ \\ ${ }^{1}$ Institute of Mathematics and Information Technologies, \\ 29, Do'rmon Yo'li str., Tashkent, 100125, Uzbekistan. \\ Email: rozikovu@yandex.ru
}

\begin{abstract}
In this note we consider several kind of partition functions of onedimensional models with nearest - neighbor interactions $I_{n}, n \in \mathbf{Z}$ and spin values \pm 1 . We derive systems of recursive equations for each kind of such functions. These systems depend on parameters $I_{n}, n \in \mathbf{Z}$. Under some conditions on the parameters we describe solutions of the systems of recursive equations.
\end{abstract}

Key words: One-dimension; configuration; partition function; recursive equation.

\section{Introduction}

The properties of physical, biological and many other systems can be described by differential and recursive equations; the latter are also called discrete dynamical system (see e.g. [2], [3], [10]). Also systems of non-linear, higher dimensional recursive equations arise in solving many different problems (see e.g. [1], [5], [6], [9],[11], [12]). But theory of the systems of recursive 
equations is not developed enough. So for each such a system one has to use a specific argument which is suitable for soling the system.

In the paper we consider the Hamiltonian (energy)

$$
H(\sigma)=\sum_{l=(x-1, x): x \in \mathbf{Z}} I_{x} \mathbf{1}_{\sigma(x-1) \neq \sigma(x)},
$$

where $\mathbf{Z}=\{\ldots,-2,-1,0,1,2, \ldots\}, \sigma$ is a function (configuration), $\sigma: \mathbf{Z} \rightarrow$ $\{-1,1\}$, (the set of all configurations $\sigma$ is denoted by $\Omega=\{-1,1\}^{\mathbf{Z}}$ ) and $I_{x} \in R$ for any $x \in \mathbf{Z}$.

In statistical physics the Hamiltonian (1) is called an one-dimensional (1D) model.

Let us consider a sequence $\Lambda_{n}=[-n, n], n=0,1, \ldots$ and denote $\Lambda_{n}^{c}=$ $\mathbf{Z} \backslash \Lambda_{n}$. Consider a boundary condition $\sigma_{n}^{(+)}=\sigma_{\Lambda_{n}^{c}}=\left\{\sigma(x)=+1: x \in \Lambda_{n}^{c}\right\}$. The energy $H_{n}^{+}(\sigma)$ of the configuration $\sigma$ in the presence of the boundary condition $\sigma_{n}^{(+)}$is expressed by the formula

$$
H_{n}^{+}(\sigma)=\sum_{l=(x-1, x): x \in \Lambda_{n}} I_{x} \mathbf{1}_{\sigma(x-1) \neq \sigma(x)}+I_{-n} \mathbf{1}_{\sigma(-n) \neq 1}+I_{n+1} \mathbf{1}_{\sigma(n) \neq 1} .
$$

The Gibbs measure on $\Omega_{n}=\{-1,1\}^{\Lambda_{n}}$ with respect to the boundary condition $\sigma_{n}^{(+)}$is defined by

$$
\mu_{n, \beta}^{+}(\sigma)=Z^{-1}(n, \beta,+) \exp \left(-\beta H_{n}^{+}(\sigma)\right),
$$

where $\beta=T^{-1}, T>0-$ temperature and $Z(n, \beta,+)$ is the normalizing factor (statistical sum or partition function):

$$
Z(n, \beta,+)=\sum_{\varphi \in \Omega_{n}} \exp \left(-\beta H_{n}^{+}(\varphi)\right) .
$$

Note that the probability (with respect to measure $\mu_{n, \beta}^{+}$) of a subset $\Omega_{n}^{\prime}$ of $\Omega_{n}$ is defined by

$$
\mu_{n, \beta}^{+}\left(\Omega_{n}^{\prime}\right)=Z^{-1}(n, \beta,+) \sum_{\psi \in \Omega_{n}^{\prime}} \exp \left(-\beta H_{n}^{+}(\psi)\right)=\frac{Z^{\prime}(n, \beta,+)}{Z(n, \beta,+)},
$$


where $Z^{\prime}(n, \beta,+)$ is called a "crystal" partition function:

$$
Z^{\prime}(n, \beta,+)=\sum_{\psi \in \Omega_{n}^{\prime}} \exp \left(-\beta H_{n}^{+}(\psi)\right)
$$

So to define the Gibbs measure and probability of an event of the system one has to compute the partition functions. If $\mu_{\beta}^{+}=\lim _{n \rightarrow \infty} \mu_{n, \beta}^{+}$exists then it is called a limit Gibbs measure. A limit Gibbs measure for a given type of interaction (energy) may fail to be unique this means that the physical system with this interaction can take several distinct equilibria i.e there is phase transition.

Note that (see [4], p.95) for the model (1) on $N=\{1,2, \ldots\}$ it was shown that there occurs a phase transition iff $\sum_{n \geq 1} e^{-2 I_{n}}<\infty$. In [8] using a contour argument it has been proven that for that model (1) the phase transition occurs if $I_{n}+I_{n+k}>k$ for any $n \in \mathbf{Z}, k \in N$.

In this paper we consider some (crystal) partition functions of the model and give the system of recursive equations for the functions. Under some conditions on parameters of the model we describe their solutions.

\section{Partition function of " + " and " $\pm "$-boundary conditions}

Consider two type of partition functions:

$$
\begin{aligned}
& Z_{n}^{+}=\sum_{\sigma_{n} \in \Omega_{n}} \exp \left\{-\beta H_{n}^{+}\left(\sigma_{n}\right)\right\}, \\
& Z_{n}^{ \pm}=\sum_{\sigma_{n} \in \Omega_{n}} \exp \left\{-\beta H_{n}^{ \pm}\left(\sigma_{n}\right)\right\},
\end{aligned}
$$

where $H_{n}^{+}$is defined by (2) and

$$
H_{n}^{ \pm}\left(\sigma_{n}\right)=H_{n}^{+}\left(\sigma_{n}\right)+I_{-n} \sigma(-n)
$$


In this paper for the simplicity assume

$$
I_{n}=I_{-n+1}, \text { for any } n \in \mathbf{Z} \text {. }
$$

Proposition 2.1. If condition (10) is satisfied then the partition functions (7) and (8) have the form

$$
\begin{aligned}
& Z_{n}^{+}=\frac{1}{2}\left(\prod_{i=0}^{n}\left(1+e^{-\beta I_{i+1}}\right)^{2}+\prod_{i=0}^{n}\left(1-e^{-\beta I_{i+1}}\right)^{2}\right), \\
& Z_{n}^{ \pm}=\frac{1}{2}\left(\prod_{i=0}^{n}\left(1+e^{-\beta I_{i+1}}\right)^{2}-\prod_{i=0}^{n}\left(1-e^{-\beta I_{i+1}}\right)^{2}\right) .
\end{aligned}
$$

Proof. Under the condition (10) we get $Z_{n}^{-}=Z_{n}^{+}$and $Z_{n}^{ \pm}=Z_{n}^{\mp}$. Now from (7), (8) we obtain the following system of recursive equations

$$
\begin{aligned}
& Z_{n}^{+}=\left(1+e^{-2 \beta I_{n+1}}\right) Z_{n-1}^{+}+2 e^{-\beta I_{n+1}} Z_{n-1}^{ \pm} \\
& Z_{n}^{ \pm}=\left(1+e^{-2 \beta I_{n+1}}\right) Z_{n-1}^{ \pm}+2 e^{-\beta I_{n+1}} Z_{n-1}^{+}
\end{aligned}
$$

Putting $X_{n}=Z_{n}^{+}-Z_{n}^{ \pm}$and $Y_{n}=Z_{n}^{+}+Z_{n}^{ \pm}$from (12) we get

$$
\begin{aligned}
& X_{n}=\left(1-e^{-\beta I_{n+1}}\right)^{2} X_{n-1}, \\
& Y_{n}=\left(1+e^{-\beta I_{n+1}}\right)^{2} Y_{n-1} .
\end{aligned}
$$

The equalities $X_{0}=Z_{0}^{+}-Z_{0}^{ \pm}=\left(1-e^{-\beta I_{1}}\right)^{2}, \quad Y_{0}=\left(1+e^{-\beta I_{1}}\right)^{2}$ with (13) imply

$$
X_{n}=\prod_{i=0}^{n}\left(1-e^{-\beta I_{i+1}}\right)^{2}, \quad Y_{n}=\prod_{i=0}^{n}\left(1+e^{-\beta I_{i+1}}\right)^{2} .
$$

Hence we get (11).

For example, in a case of the usual Ising model i.e. $I_{m}=I, \forall m \in \mathbf{Z}$ from (11) denoting $\tau=\exp (-\beta I)$ we get

$$
\begin{aligned}
& Z_{n}^{+}=\frac{1}{2}\left((1+\tau)^{2(n+1)}+(1-\tau)^{2(n+1)}\right), \\
& Z_{n}^{ \pm}=\frac{1}{2}\left((1+\tau)^{2(n+1)}-(1-\tau)^{2(n+1)}\right) .
\end{aligned}
$$


Using these equalities (for usual Ising model) it is easy to see that

$$
\frac{Z_{n}^{+}}{Z_{n}^{ \pm}} \rightarrow 1, \text { if } n \rightarrow \infty
$$

This means that for the Ising model the partition functions $Z_{n}^{+}$and $Z_{n}^{ \pm}$are asymptotically equal. This gives in fact uniqueness of limit Gibbs measure for the 1D Ising model. Such an asymptotical equality is true if $I_{m}$ is a periodic function of $m$ i.e $I_{m+p}=I_{m}$ for some $p \geq 1$ and all $m \in N$.

\section{Crystal partition functions}

In this section we are going to describe the crystal partition functions.

Denote $\Omega_{m, n}=\{-1,1\}^{[m, n]}$, where $[m, n]=\{m, m+1, \ldots, n\}, m, n \in$ $\mathbf{Z}, n \geq m$. Put

$$
N_{\varepsilon}(\sigma)=|\{x \in[m, n]: \sigma(x)=\varepsilon\}|, \varepsilon= \pm 1,
$$

where $|S|$ is the cardinal of the set $S$. For $r=0,1, \ldots, n-m+1$ consider the following crystal partition functions:

$$
\begin{gathered}
Z_{m, n}^{\varepsilon, r}=\sum_{\sigma \in \Omega_{m, n}: N_{-\varepsilon}(\sigma)=r} e^{-\beta H^{\varepsilon}(\sigma)}, \quad \varepsilon=-,+ \\
Z_{m, n}^{ \pm, r}=\sum_{\sigma \in \Omega_{m, n}: N_{+}(\sigma)=r} e^{-\beta H^{ \pm}(\sigma)} .
\end{gathered}
$$

Note that $Z_{m, n}^{-, r}=Z_{m, n}^{+, r}$. Denoting $X_{m, n}^{r}=Z_{m, n}^{-, r}$ and $Y_{m, n}^{r}=Z_{m, n}^{ \pm, r}$ from (14) and (15) one easily gets the following system of (multi-variable) recursive equations

$$
\begin{aligned}
& X_{m, n}^{r}=X_{m, n-1}^{r-1}+e^{-\beta I_{n+1}} Y_{m, n-1}^{r}, \\
& Y_{m, n}^{r}=Y_{m, n-1}^{r}+e^{-\beta I_{n+1}} X_{m, n-1}^{r-1},
\end{aligned}
$$

where $r=0,1, \ldots, n-m+1, m, n \in \mathbf{Z}, n \geq m$. 
The system (16) can be reduced to a recursive equation with respect to $X_{m, n}^{r}$. Indeed, from the first equation of (16) we get

$$
Y_{m, n-1}^{r}=e^{\beta I_{n+1}}\left(X_{m, n}^{r}-X_{m, n-1}^{r-1}\right)
$$

Now from the second equation of (16) using (17) we get

$$
X_{m, n}^{r}=X_{m, n-1}^{r-1}+e^{-\beta\left(I_{n+1}-I_{n}\right)}\left(X_{m, n-1}^{r}-X_{m, n-2}^{r-1}\right)+e^{-\beta\left(I_{n}+I_{n+1}\right)} X_{m, n-2}^{r-1},
$$

where $r=1,2, \ldots, n-m+1, n \geq m$ and

$$
\begin{gathered}
X_{m, m}^{0}=1, \quad X_{m, m}^{1}=e^{-\beta\left(I_{m}+I_{m+1}\right)}, \quad X_{m, m+1}^{0}=1, \\
X_{m, m+1}^{1}=e^{-\beta\left(I_{m}+I_{m+1}\right)}+e^{-\beta\left(I_{m+1}+I_{m+2}\right)}, \quad X_{m, m+1}^{2}=e^{-\beta\left(I_{m}+I_{m+2}\right)}, m \in \mathbf{Z} .
\end{gathered}
$$

Iterating (18) one can obtain an expression for $X_{m, n}^{r}$. Then using (17) one can find $Y_{m, n}^{r}$. But these expressions would be in a very bulky form.

Now we shall illustrate such an expression for the Ising model i.e. $I_{m} \equiv$ $I, m \in \mathbf{Z}$. In this case the recurrence equation (18) becomes more simple

$$
X_{n}^{r}=X_{n-1}^{r-1}+X_{n-1}^{r}+(\chi-1) X_{n-2}^{r-1}
$$

where $X_{n}^{r}=X_{m, n_{1}}^{r}$ with $n_{1}-m=n, \chi=e^{-2 \beta I}$.

It is easy to see that for $r=0,1,2,3,4$ the solutions are

$$
\begin{gathered}
X_{n}^{0}=1, \quad X_{n}^{1}=n \chi, \quad X_{n}^{2}=(n-1) \chi+\frac{(n-2)(n-1)}{2 !} \chi^{2}, \\
X_{n}^{3}=(n-2) \chi+(n-2)(n-3) \chi^{2}+\frac{(n-2)(n-3)(n-4)}{3 !} \chi^{3}, \\
X_{n}^{4}=(n-3) \chi+\frac{3(n-3)(n-4)}{2} \chi^{2}+\frac{(n-3)(n-4)(n-5)}{2} \chi^{3}+ \\
\frac{(n-3)(n-4)(n-5)(n-6)}{4 !} \chi^{4},
\end{gathered}
$$


here we used the following formulas

$$
\sum_{j=1}^{n} j^{2}=\frac{1}{6}\left(2 n^{3}+3 n^{2}+n\right), \quad \sum_{j=1}^{n} j^{3}=\frac{1}{4}\left(n^{4}+2 n^{3}+n^{2}\right) .
$$

Note that $X_{n}^{r}$ has a form

$$
X_{n}^{r}=a_{1, n}^{r} \chi+a_{2, n}^{r} \chi^{2}+\ldots+a_{r, n}^{r} \chi^{r} .
$$

For the coefficients $a_{k, n}^{r}, 0 \leq k \leq r \leq n$, using (19) we obtain the following system of recursive equations

$$
\begin{aligned}
& a_{1, n}^{r}=a_{1, n-1}^{r}+a_{1, n-1}^{r-1}-a_{1, n-2}^{r-1}, \\
& a_{k, n}^{r}=a_{k, n-1}^{r}+a_{k, n-1}^{r-1}+a_{k-1, n-2}^{r-1}-a_{k, n-2}^{r-1}, \quad k=2,3, \ldots, r-1, \\
& a_{r, n}^{r}=a_{r, n-1}^{r}+a_{r-1, n-2}^{r-1},
\end{aligned}
$$

The following lemma gives solution to (20)

Lemma 3.1. Solution of the system of recursive equations (20) is

$$
a_{k, n}^{r}=\left(\begin{array}{c}
n-r+1 \\
k
\end{array}\right)\left(\begin{array}{l}
r-1 \\
k-1
\end{array}\right), \quad 0 \leq k \leq r \leq n .
$$

Proof. We shall use mathematical induction (cf. with [7] pages 148-150). Let $A_{m}$ denote all cases of (21) with $n+k+r=m$. Formulas given above for $X_{n}^{r}, \quad r=0,1,2,3,4$ show that the formula (21) is true for small values of $m$. Assuming that $A_{m}$ holds, we are to prove $A_{m+1}$ that is, equation (20), for any integers $n, r$ and $k$ whose sum is $m+1$. Since RHS of equation (20) contains terms with $n+r+k \leq m$ using the assumption of the induction for each term of RHS of (20) we get (21).

Thus the solution of (19) is given by

$$
X_{n}^{r}=\sum_{k=1}^{r}\left(\begin{array}{c}
n-r+1 \\
k
\end{array}\right)\left(\begin{array}{l}
r-1 \\
k-1
\end{array}\right) \chi^{k}
$$


Remark 1. Note that for $\chi=1$ (i.e. there is no interaction) the solution of (19) is $X_{n}^{r}=\left(\begin{array}{l}n \\ r\end{array}\right)$. Using (22) for $\chi=1$ we obtain the following property of binomial coefficients

$$
\left(\begin{array}{l}
n \\
r
\end{array}\right)=\sum_{k=1}^{r}\left(\begin{array}{c}
n-r+1 \\
k
\end{array}\right)\left(\begin{array}{l}
r-1 \\
k-1
\end{array}\right) .
$$

This identity is known as the convolution identity of Vandermonde.

Since interaction (parameter $I$ ) of the 1D Ising model is translationinvariant (does not depend on the points of $\mathbf{Z}$ ), the unknown functions $X_{m, n}^{r}, Y_{m, n}^{r}$ of the system (16) depend on $n-m$ and $r$ only (see (19)), consequently, instate of $n-m$ we can write $n$. Summarizing the results for the Ising model we have

Theorem 3.1. For the Ising model the solution of the system of recursive equations (16) is

$$
\begin{gathered}
X_{n}^{r}=\sum_{k=1}^{r}\left(\begin{array}{c}
n-r+1 \\
k
\end{array}\right)\left(\begin{array}{l}
r-1 \\
k-1
\end{array}\right) \chi^{k}, \\
Y_{n}^{r}=\frac{1}{\sqrt{\chi}}\left(X_{n+1}^{r}-X_{n}^{r-1}\right),
\end{gathered}
$$

where $n$ stands for $n-m$.

Acknowledgments. I thank the Abdus Salam International Center for Theoretical Physics (ICTP), Trieste, Italy for providing financial support of my visit to ICTP (February-April 2009).

\section{References}

[1] P. D'Alessandro, M. Dalla Mora, Systems, memory, causality, evolution and recursive equations, Comp. Maths with Appl. 10 (1984) 61-69. 
[2] R.L. Devaney, An introduction to chaotic dynamical system, Westview Press, 2003.

[3] N.N. Ganikhodjaev, U.A. Rozikov, On quadratic stochastic operators generated by Gibbs distributions, Regul. Chaotic Dynamics 11 (2006) 467-473.

[4] H-O. Georgii, Gibbs Measures and Phase Transitions, Walter de Gruyter, Berlin, 1988.

[5] N. K. Krivulin, A recursive equations based representation for the G/G/m queue, Appl. Math. Lett. 7 (1994) 73-77.

[6] F.M. Mukhamedov, On a recursive equation over p-adic field, Appl. Math. Lett. 20 (2007) 88-92.

[7] I. Niven, Mathematics of Choice, The Math. Assoc. America. V.15. 1965.

[8] U.A. Rozikov, An example of one-dimensional phase transition, Siber. Adv. Math. 16 (2006) 121-125.

[9] U.A. Rozikov, On countably periodic Gibbs measures of the Ising model on the Cayley tree, Theor. Math. Phys. 130 (2002) 92-100.

[10] A.P. Shapiro, S.P. Luppov, Recursive equations in the theory of population biology, Nauka, Moscow, 1983.(Russian)

[11] Y.M. Suhov, U.A. Rozikov, A hard-core model on a Cayley tree: an example of a loss network, Queueing Syst. 46 (2004) 197-212.

[12] N. Tanimura, O. Tanimura, Recursive equation of character expansion coefficients in lattice gauge theory, J. Math. Phys. 32 (1991) 1928-1933. 